\title{
Systems level expression correlation of Ras GTPase regulators
}

\author{
E. Besray Unal ${ }^{1,2 \dagger}$, Christina Kiel ${ }^{3,4^{*}+} \mathbb{D}$, Hannah Benisty ${ }^{3}$, Andrew Campbell ${ }^{5}$, Karen Pickering ${ }^{5}$, Nils Blüthgen ${ }^{1,2}$, \\ Owen J. Sansom ${ }^{5}$ and Luis Serrano $0^{3,6,7^{*}}$
}

\begin{abstract}
Background: Proteins of the ubiquitously expressed core proteome are quantitatively correlated across multiple eukaryotic species. In addition, it was found that many protein paralogues exhibit expression anticorrelation, suggesting that the total level of protein with a given functionality must be kept constant.

Methods: We performed Spearman's rank correlation analyses of gene expression levels for the RAS GTPase subfamily and their regulatory GEF and GAP proteins across tissues and across individuals for each tissue. A large set of published data for normal tissues from a wide range of species, human cancer tissues and human cell lines was analysed.

Results: We show that although the multidomain regulatory proteins of Ras GTPases exhibit considerable tissue and individual gene expression variability, their total amounts are balanced in normal tissues. In a given tissue, the sum of activating (GEFs) and deactivating (GAPs) domains of Ras GTPases can vary considerably, but each person has balanced GEF and GAP levels. This balance is impaired in cell lines and in cancer tissues for some individuals.

Conclusions: Our results are relevant for critical considerations of knock out experiments, where functionally related homologs may compensate for the down regulation of a protein.
\end{abstract}

Keywords: Ras small GTPases, Tissue expression, Gene expression network, GTPase activating proteins, Guanine nucleotide exchange factors

\section{Background}

The systematic profiling of gene and protein expression levels in different tissues and cell types has enabled the definition of common and unique components and the functional characterisation of tissues and organs ('What makes a cell type?') [1-4]. Expression and clustering analysis revealed that core conserved genes and proteins are expressed at similar levels in different eukaryotes [5] and across various mammalian and non-mammalian vertebrate tissues $[6,7]$. However, it is unclear if there is a quantitative expression association for groups of functionally related genes and family members (homologs) that do not belong to the evolutionary conserved ubiquitous expressed core genes and proteins. Recently it has

\footnotetext{
* Correspondence: christina.kiel@ucd.ie; luis.serrano@crg.es; luis.serrano@crg.eu

${ }^{\dagger}$ E. Besray Unal and Christina Kiel contributed equally to this work.

${ }^{3}$ Centre for Genomic Regulation (CRG), Systems Biology Programme. The Barcelona Institute of Science and Technology, Dr. Aiguader 88, Barcelona 08003, Spain

Full list of author information is available at the end of the article
}

been shown that functional divergence of paralogs is fast, promoting tissue specificity [8]; in this, orthologues show a high conservation in tissue-specificity, whilst paralogs show less conservation [9]. We have shown previously that paralogous gene pairs that are less similar in sequence homology and domain composition showed expression anticorrelation profiles during the calcium-induced differentiation of primary human keratinocytes [10]. Anticorrelation of paralog gene expression was also observed in different brain regions [11]. This suggests specialized time- and space-dependent roles of different paralogs and, more importantly, the need to keep the same level of common functionalities carried out by paralogs. We could ask if the same is true for antagonistic activities regulating essential cell processes. An interesting question is if the same could happen for antagonistic activities regulating essential cell functions, such as those mediated by the family of small GTPase proteins.

To answer this question about a family-wide expression balance, we choose the Ras p21 subfamily of small 
GTPases [12] (associated with cancer [13]), together with their regulators, which direct diverse cellular processes by cycling between GTP-bound active and GDP-bound inactive conformations (Fig. 1a) [13, 14]. Cycling between the 'OFF states' (GDP-bound) and the 'ON-states' (GTPbound) of Ras proteins is catalysed GTP exchange factors domains (RasGEFs) and GTP activating domains
(RasGAP). The GEF and GAP regulators are multidomain proteins of diverse composition (e.g. SH2, SH3, PH, RBD domains) in addition to their catalytic GEF or GAP domains (Additional file 1: Figure S1). Because each RAS subfamily has GEFs and GAPs with unique catalytic domains of 3D structural and sequence similarity, this makes them in principle specific for each respective subfamily [15-17].

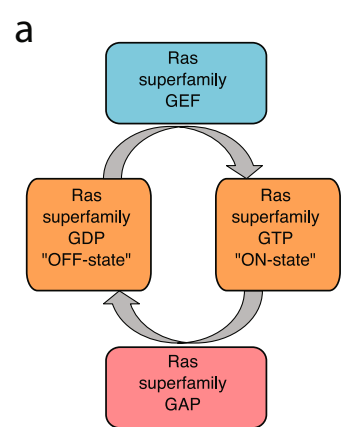

C

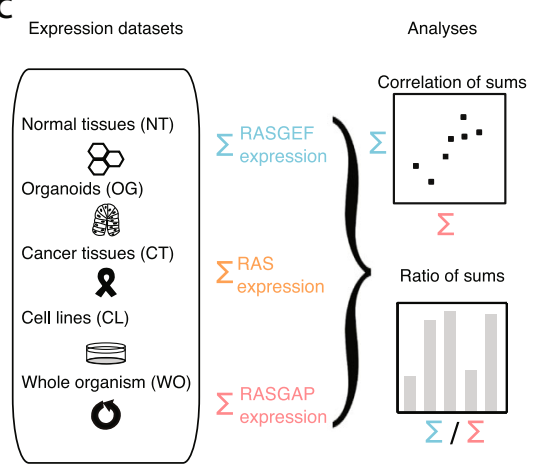

e

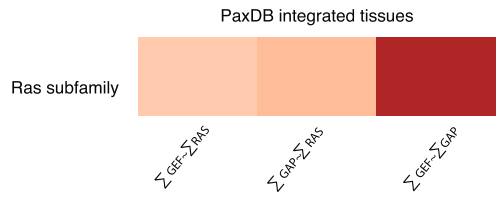

$f$

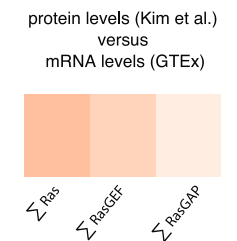

b

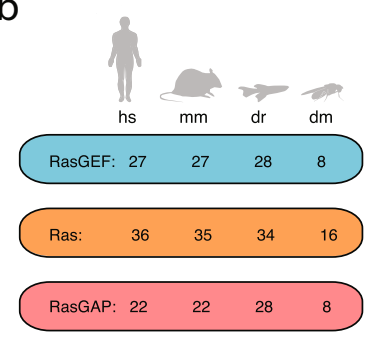

d
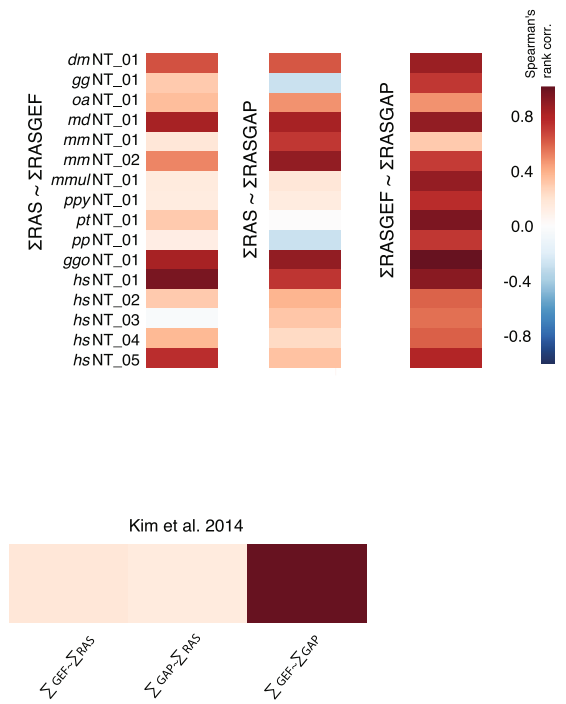

Fig. 1 The Ras subfamily and their regulators, overview of analyses performed, and Spearman's rank correlations. a Schematic diagram of the Ras superfamily of small GTPases that cycle between a GDP-bound "OFF-state" and a GTP-bound "ON-state". This cycling is regulated by GEF and GAP proteins. b Number of Ras subfamily and regulators genes in four species (Homo sapiens, hs; Mus musculus, mm; dr, Danio rerio, dr, Drosophila melanogaster, $\mathrm{dm}$ ). c Schematic figure of protein and gene expression datasets used, and main data analyses performed in this study. $\mathbf{d}$ Spearman's rank correlation values of the $\sum$ RAS vs $\sum G E F, \sum R A S$ vs $\sum G A P$, and the $\sum$ GEF vs $\sum G A P$ displayed as a heat map (normal tissue datasets dmNT_01, ggNT_01, oaNT, mdNT_01, mmNT_01, mmNT_02, mmuNT_01, ppyNT_01, ptNT_01, ppNT_01, ggoNT_01, hsNT_01, hsNT_02, hsNT_03, hsNT_04, hsNT_05). e Heatmap representation of Spearman's rank correlations of protein levels for GEFs with RASs, GAPs with RASs, and GEFs with GAPs across human normal tissues. While correlations between the GEF with the RAS and the GAP with the RAS were generally poorer, higher correlations between the GEF with the GAP were observed independently in both proteomic datasets. $\mathbf{f}$ Heatmap representation of Spearman's rank correlations of protein and RNA levels (hsNT_03). Parts of the pictures were drawn using Inkscape 
Here, we have analysed the gene and protein expression levels for the RAS GTPase subfamily and their regulatory GEF and GAP proteins using a large set of published data for normal tissues from a wide range of species, human cancer tissues and human cell lines. We show that the sum of all GEF family members ( $\sum$ RASGEF) generally correlates with the sum of all GAP family members ( $2 \mathrm{RASGAP}$ ) in physiological normal tissues, although the total sums for GEFs and GAPs could change significantly between individuals. Thus, there is a balance in gene and protein expression levels of RAS regulators for normal tissues. These $\Sigma$ RASGEF- $\sum$ RASGAP correlations are lost or have a different slope in transformed cell lines and in some individual cancer tissues. This is caused by a higher sum of GAP levels in cancer tissues of some individuals. Our work shows that to understand the functionality of cell processes it is important to consider all members of a protein family. Individual proteins levels can be different from tissue to tissue, but the sum of homologs is constant, possibly to ensure adequate functional balance.

\section{Methods}

\section{Transcriptomics data sources}

We collected the following published datasets: ( $\left.h s \mathrm{NT} \_01\right)$ RNA-Seq gene expression dataset associated to $H$. sapiens 11 normal tissues [18] ( $h s$ NT_02) RNA-Seq gene expression dataset associated to $H$. sapiens 32 normal tissues (https://www.ebi.ac.uk/arrayexpress/experiments/E-MTAB -2836/); ( $h s$ NT_03) RNA-Seq gene expression dataset associated to $H$. sapiens 53 normal tissues [19, 20] (GTEx); ( $h s \mathrm{NT}$ _04) RNA-Seq gene expression dataset associated to H. sapiens 18 normal tissues (TCGA Research Network, http://cancergenome.nih.gov/); (hsNT_05) RNA-Seq gene expression dataset associated to $H$. sapiens 8 tissues [21]; (ggoNT_01) RNA-Seq gene expression dataset associated to G. gorilla 6 tissues [21]; (ptNT_01) RNA-Seq gene expression dataset associated to P. troglodytes 6 tissues [21]; ( $p p$ NT_01) RNA-Seq gene expression dataset associated to $P$. paniscus 6 tissues [21]; (ppyNT_01) RNA-Seq gene expression dataset associated to $P$. pygmaeus 5 tissues [21]; (mmulNT_01) RNA-Seq gene expression dataset associated to $M$. mulatta 7 tissues [21]; (mmNT_01) RNA-Seq gene expression dataset associated to M. musculus 6 tissues [21]; ( $\left.m d \mathrm{NT}_{-} 01\right)$ RNA-Seq gene expression dataset associated to $M$. domestica 6 tissues [21]; (oaNT_01) RNA-Seq gene expression dataset associated to O. anatinus 6 tissues [21]; (ggNT_01) RNA-Seq gene expression dataset associated to G. gallus 6 tissues [21]; (mmNT_02) microarray gene expression dataset associated to $M$. musculus 45 normal tissues [22]; $\left(d m \mathrm{NT} \_01\right)$ microarray gene expression dataset associated to D. melanogaster 25 normal tissues [23]; (hsCT_01) RNA-Seq gene expression dataset associated to H. sapiens 18 cancer tissues (TCGA Research Network http://cancergenome.nih.gov/); (hsCT_02) microarray gene expression dataset associated to $H$. sapiens 31 cancer tissues [24] (COSMIC sanger cell line project); (hsCT_03) microarray gene expression dataset associated to $H$. sapiens 163 cancer tissues (Expo database, http://www.intgen.org/ research-services/biobanking-experience/expo/); (hsCL_01) RNA-Seq gene expression dataset associated to $H$. sapiens 56 cell lines (Human Protein Atlas, http://www.ncbi.nlm.nih.gov/bioproject/PRJNA183192); (hsCTL_02) microarray gene expression dataset associated to $H$. sapiens cell lines from 24 cancer tissues (Cancer Cell Line Encyclopedia [25]). RPKM (Reads Per Kilobase per Million mapped reads) or TPM (Transcripts Per Kilobase Million) values were used for RNA-Seq data, while total RNA levels from microarray datasets were utilized. We have always considered the most expressed isoform for each gene.

\section{Proteomics data sources}

The PaxDB, a mass spectrometry-based proteomic meta-resource across organisms was used to extract protein information [26]. The average spectral counting value derived from all experiments (in different cell lines and tissues) corresponds to an approximate protein abundance estimate for the respective organism (ppm values for 'whole organisms' and integrated tissues' in PaxDb). To retrieve protein levels for RAS, GEF, and GAP members in different organisms and under different conditions (e.g. cell lines, normal tissues), we first analyzed the PaxDB (Additional file 2). We found a good coverage of expression information using the 'whole organism' expression score, which is an average spectral counting value derived from all mass spectrometry experiments from different cell lines and tissues. Similarly, we retrieved integrated expression information for 20 tissues from the PaxDB and for 20 tissues from a recent deep proteomic study [3]. However, the complete quantification of lower abundance proteins and of isoforms and family members (that often contain only a few unique or no unique tryptic peptides) by mass spectrometry in tissues is not feasible [27].

\section{Selection of Ras, RasGEF, and RasGAP family members}

Members of the Ras family were selected based on the presence of a predicted Ras domain using either the SMART (accession number: SM00173; http://smart.embl-heidelberg.de/) or the Pfam database (accession number: PF00071; https://pfam.xfam.org/) (Table 1, Table 2, Additional file 3). All 36 Ras family members were also present in a detailed bioinformatics evolutionary tree analysis of the whole RAS superfamily [12]. Members of the RasGEF family were selected based on the presence of a predicted Ras domain using either the SMART (accession number: SM00147; http://smart.embl-heidelberg.de/) or the Pfam database (accession 
Table 1 Selection of Ras superfamily members. Selection of family members based on domain predictions using the SMART or Pfam databases, or based on Rojas et al., 2012

\begin{tabular}{|c|c|c|c|c|}
\hline & Gene name & $\begin{array}{l}\text { RAS domain } \\
\text { in SMART }\end{array}$ & $\begin{array}{l}\text { RAS domain } \\
\text { in Pfam }\end{array}$ & Rojas et al. 2012 \\
\hline \multirow[t]{36}{*}{ Ras family } & DIRAS1 & YES & YES & YES \\
\hline & DIRAS2 & YES & YES & YES \\
\hline & DIRAS3 & NO & YES & YES \\
\hline & ERAS & YES & YES & YES \\
\hline & GEM & NO & YES & YES \\
\hline & HRAS & YES & YES & YES \\
\hline & KRAS & YES & YES & YES \\
\hline & MRAS & YES & YES & YES \\
\hline & NKIRAS1 & NO & YES & YES \\
\hline & NKIRAS2 & NO & YES & YES \\
\hline & NRAS & YES & YES & YES \\
\hline & RALA & YES & YES & YES \\
\hline & RALB & YES & YES & YES \\
\hline & RAP1A & YES & YES & YES \\
\hline & RAP1B & YES & YES & YES \\
\hline & RAP2A & YES & YES & YES \\
\hline & RAP2B & YES & YES & YES \\
\hline & RAP2C & YES & YES & YES \\
\hline & RASD1 & YES & YES & YES \\
\hline & RASD2 & YES & YES & YES \\
\hline & RASL10A & NO & YES & YES \\
\hline & RASL10B & NO & YES & YES \\
\hline & RASL11A & NO & YES & YES \\
\hline & RASL11B & NO & YES & YES \\
\hline & RASL12 & NO & YES & YES \\
\hline & REM1 & NO & YES & YES \\
\hline & REM2 & NO & YES & YES \\
\hline & RERG & YES & YES & YES \\
\hline & RERGL & NO & YES & YES \\
\hline & RHEB & YES & NO & YES \\
\hline & RHEBL1 & YES & NO & YES \\
\hline & RIT1 & YES & NO & YES \\
\hline & RIT2 & YES & NO & YES \\
\hline & RRAD & NO & YES & YES \\
\hline & RRAS & YES & YES & YES \\
\hline & RRAS2 & YES & YES & YES \\
\hline
\end{tabular}

number: PF00617; https://pfam.xfam.org/). Members of the RasGAP/[RapGAP] family were selected based on the presence of a predicted RasGAP domain using either the SMART (accession number: SM00323; http://smart.embl-heidelberg.de/) or the Pfam database (accession numbers RasGAP: PF00616 and RapGAP: PF02145; https://pfam.xfam.org/). NF2 was excluded from the list of RasGAPs as despite its name, NF2 is not related to NF1 and does not display GAP activity against any Ras GTPase [15].

\section{Gene expression values and sums}

The data sets labeled as $m m \mathrm{NT} \_02, d m \mathrm{NT} \_01, h s \mathrm{CT} \_02$, $h s \mathrm{CT} \_03$, and $h s \mathrm{CL} \_02$ are based on microarray measurements. For these data sets, total RNA levels were utilized. The remaining data sets are based on RNA sequencing technology. The RPKM ( $h s \mathrm{NT}_{-} 01, h s \mathrm{NT}$ 03, $h s \mathrm{NT}$-04, $h s \mathrm{NT} \_05, g g o \mathrm{NT} \_01, p t \mathrm{NT} \_01, p p \mathrm{NT}$ 01, ppyNT_01, mmulNT_01, mmNT_01, $m d \mathrm{NT} \_01, o a \mathrm{NT} \_01, g g \mathrm{NT} \_01$, $\left.h s \mathrm{CT} \_01\right)$ or TPM (hsNT_02, $\left.h s \mathrm{CL} \_01\right)$ values (the way as presented by the original studies/databases) were utilized without applying any normalization or scaling factor. The sums for RAS, GEF, and GAP genes were computed by summing the original data for each corresponding gene. If there was more than one splice variant for a gene, the maximum expression variant was used. There are some genes missing in some of the data sets (especially in microarray-based sets). The missing gene expression level was assumed to be zero.

\section{Correlations}

The gene expressions (array-based, RPKM or TPM) were used to determine the sum of GEF and GAP members, in different tissues/cell models with the Spearman's rank correlation metric. Random correlations were determined based on independent shuffling of the expression levels of Ras family genes. The procedure was repeated 10,000 times, for each dataset. Finally, the real correlation value was compared to the distribution of shuffled correlations. The hypothesis that the real correlation value is significantly different than the random correlation distribution was tested via $p$-value, based on $\mathrm{z}$-scores. To check that the high correlations were not dominated by one highly expressed family member, each GEF and GAP was removed one by one and the correlation was re-calculated ('bootstrapping'). The effect of gene removal on GEF to

GAP correlation values was not significant for either the normal tissue or the cancer tissue datasets. The significance of the difference between the matching normal and cancer dataset correlation values was determined by Fisher's test; the correlation values were transformed to $\mathrm{z}$-scores by using the sample size (number of individuals).

\section{Ratios}

The gene expression levels from TCGA data set were used to determine the sum of GEF and GAP members, for the matching normal and cancer tissues. Mann-Whitney $U$ test was applied to determine if the normal tissue ratios were significantly different from those of cancer tissues. 
Table 2 Selection of Ras superfamily member RasGEF and RasGAP regulators. Selection of family members based on domain predictions using the SMART or Pfam databases

\begin{tabular}{|c|c|c|c|}
\hline & Gene name & $\begin{array}{l}\text { RAS domain } \\
\text { in SMART }\end{array}$ & $\begin{array}{l}\text { RAS domain } \\
\text { in Pfam }\end{array}$ \\
\hline \multirow[t]{27}{*}{ RasGEF family } & KNDC1 & YES & YES \\
\hline & PLCE1 & YES & YES \\
\hline & RALGDS & YES & YES \\
\hline & RALGPS1 & YES & YES \\
\hline & RALGPS2 & YES & YES \\
\hline & RAPGEF1 & YES & YES \\
\hline & RAPGEF2 & YES & YES \\
\hline & RAPGEF3 & YES & YES \\
\hline & RAPGEF4 & YES & YES \\
\hline & RAPGEF5 & YES & YES \\
\hline & RAPGEF6 & YES & YES \\
\hline & RAPGEFL1 & YES & YES \\
\hline & RASGEF1A & YES & YES \\
\hline & RASGEF1B & YES & YES \\
\hline & RASGEF1C & YES & YES \\
\hline & RASGRF1 & YES & YES \\
\hline & RASGRF2 & YES & YES \\
\hline & RASGRP1 & YES & YES \\
\hline & RASGRP2 & YES & YES \\
\hline & RASGRP3 & YES & YES \\
\hline & RASGRP4 & YES & YES \\
\hline & RGL1 & YES & YES \\
\hline & RGL2 & YES & YES \\
\hline & RGL3 & YES & YES \\
\hline & RGL4 & YES & YES \\
\hline & SOS1 & YES & YES \\
\hline & SOS2 & YES & YES \\
\hline \multirow[t]{15}{*}{ RasGAP family } & DAB2IP & YES & YES \\
\hline & GARNL3 & NO & YES \\
\hline & NF1 & YES & YES \\
\hline & PLXNB1 & NO & YES \\
\hline & RALGAPA1 & NO & YES \\
\hline & RALGAPA2 & NO & YES \\
\hline & RAP1GAP & NO & YES \\
\hline & RAP1GAP2 & NO & YES \\
\hline & RASA1 & YES & YES \\
\hline & RASA2 & NO & YES \\
\hline & RASA3 & NO & YES \\
\hline & RASA4 & NO & YES \\
\hline & RASAL1 & NO & YES \\
\hline & RASAL2 & YES & YES \\
\hline & RASAL3 & YES & YES \\
\hline
\end{tabular}

Table 2 Selection of Ras superfamily member RasGEF and RasGAP regulators. Selection of family members based on domain predictions using the SMART or Pfam databases (Continued)

\begin{tabular}{llll}
\hline Gene name & $\begin{array}{l}\text { RAS domain } \\
\text { in SMART }\end{array}$ & $\begin{array}{l}\text { RAS domain } \\
\text { in Pfam }\end{array}$ \\
\hline SIPA1 & NO & YES \\
SIPA1L1 & NO & YES \\
SIPA1L2 & NO & YES \\
SIPA1L3 & NO & YES \\
SYNGAP1 & YES & YES \\
TSC2 & NO & YES \\
\hline
\end{tabular}

\section{Slope test}

The gene expression values (array-based, RPKM, or TPM) were used to determine the sum of GEF and GAP members' expression, for the matching normal and cancer tissues/ cell models. A non-parametric t-test (based on the regression line fitted to GEF GAP sums, the standard error of regression, the standard deviation of ¿GEF $\Sigma$ GAP, and the number of individuals) was used to determine the significant changes between the slopes of the matching normal and cancer samples.

$b_{n}$ : slope of the fitted line for normal tissue.

$b_{c}$ : slope of the fitted line for cancer tissue.

Syx $_{\mathrm{n}}$ : standard error of the fitted line for normal tissue.

Syx $_{\mathrm{C}}$ : standard error of the fitted line for cancer tissue $\mathrm{Sx}_{\mathrm{n}}$ : standard deviation of the sum of GEF values in normal tissue.

$\mathrm{Sx}_{\mathrm{C}}$ : standard deviation of the sum of GEF values in cancer tissue

$$
\begin{aligned}
S b_{n} & =\frac{S y x_{n}}{S x_{n}{ }^{*}\left(t_{n}-1\right)^{2}} \\
S b_{c} & =\frac{S y x_{c}}{S x_{c}{ }^{*}\left(t_{c}-1\right)^{2}}
\end{aligned}
$$

where $t_{n}$ and $t_{c}$ are the number of individuals (data points) in normal and cancer tissue data sets, respectively.

$$
\begin{aligned}
& S=S b_{n}-S b_{c} \\
& T=\left(b_{n}-b_{c}\right) / S \\
& D F=t_{n}-t_{c}-4
\end{aligned}
$$

The corresponding $p$-value for the change in the slopes for normal versus cancer tissue is determined via $\mathrm{t}$-test using the $\mathrm{T}$ and DF (degrees of freedom) values.

\section{Survival test}

The TCGA data set was employed to determine the survival rates of patients with respect to their $\sum$ RasGAP/ $\sum$ RasGEF ratios. The "TCGA2STAT" $\mathrm{R}$ library was employed to download the matching RNA-Seq (RPKM) and clinical data sets. The clinical data were utilized to 
derive the "survival time" and "death/alive status" for each patient. The gene expression values were used to determine the sum of GEF and GAP members, and the corresponding ratio for each patient. For each cancer tissue, the corresponding normal tissue data set (TCGA) was used to determine ratio thresholds; "mean of normal ratio +2 times standard deviation of normal ratio". These tissue-specific ratio thresholds were used to stratify each patient as having a high- or a low-ratio. A survival function was fitted on the stratified data (based on high- or low-ratio), survival time, and death/alive status. The Kaplan-Meier plots for these fitted survival functions were depicted. The significance of difference between high- and low-ratio values are indicated on plots, with a $\mathrm{p}$-value. The "survminer" $\mathrm{R}$ library was used for the survival analysis.

\section{Results}

\section{Correlation of $\Sigma$ RAS, $\Sigma$ RASGEF and LRASGAP levels in adult normal tissues}

We included in our study all RAS members and their GEF and GAP regulator members from human (Homo sapiens), other primates (Gorilla gorilla, Macaca mulatta, Pan paniscus, Pan troglodytes, and Pongo pygmaeus), other mammals (Mus musculus, Monodelphis domestica, and Ornithorhynchus anatinus), bird (Gallus gallus), fish (Danio rerio), and fly (Drosophila melanogaster), we used Pfam domain predictions and additional manual annotations based on recent reviews (Additional file 3) [12, 15].

We used public repositories and selected publications to retrieve gene expression data required for the subsequent workflow of performing correlations and ratios of RAS/ RASGEF/ RASGAP sums (Fig. 1c). The gene expression levels for RAS, RASGEF, and RASGAP members under different conditions (normal tissues and organs from various species, human cancer tissues, human cell lines) were retrieved and quantitative RNA Seq datasets were prioritised [28] (see Methods and Additional file 1: Figure S2). We analysed the gene expression correlations of the sums of RAS and regulators in adult fly (dmNT_01), chicken (ggNT_01), platypus (oaNT), opossum (mdNT_01), mouse (mmNT_01, $\left.m m \mathrm{NT} \_02\right)$, primates $\left(m m u \mathrm{NT} \_01, p p y \mathrm{NT} \_01, p t \mathrm{NT} \_01\right.$, $p p$ NT_01, ggoNT_01), and human (hsNT_01, hsNT_02, $h s \mathrm{NT}$ 03, hsNT_04, $h s \mathrm{NT} \_05$ ) tissues (see Additional file 4). We found good correlations between the $\Sigma$ GEF with the $\Sigma$ GAP (Fig. 1d; Additional file 1: Figure S3A). Correlations between the $\Sigma$ GEF with the $\Sigma$ RAS and the $\Sigma$ GAP with the $\Sigma$ RAS were generally poorer (Fig. 1d). While a strong and significant $(p$-value $<0.05)$ correlation between the GEFs and the GAPs across tissues was found, the individual GEFs and GAPs showed a large distribution of correlation values (Additional file 1: Figure S3B), without any bias of higher correlations being associated to higher expression values (Additional file 1: Figure S3C). Thus, while the gene expression levels of individual GEF and GAP family members may vary in a given tissue, the sum of all GEFs and GAPs for a RAS subfamily is correlated.

To measure the significance of the correlation values, the RAS, GEF, and GAP gene expression values were shuffled 10,000 times and the correlation between the $\Sigma$ GEFs and the $\Sigma$ GAPs was determined (Additional file 5). We observed a strong correlation for the $\Sigma$ GEF and the $\Sigma$ GAP levels across normal adult tissues in most data in comparison to the shuffled background ( $p$-value $<0.05$ ) (Additional file 1: Figure S4). Focusing on a subset of experimentally validated GEFs and GAPs (RASGEF1A, RASGRP1, RASGRP4, SOS1, RASGRP2, NF1, RASA1, and RASA4) identified significant, but lower correlations values in all five human normal datasets (Additional file 5).

We also confirmed the GEF to GAP gene expression correlation across normal tissues at the protein level using the spectral counting score of the mass spectrometry-based proteomic meta-resource PaxDB [26]. The whole organism $\Sigma$ RAS, $\Sigma$ RASGEF and $\Sigma$ RASGAP protein levels for human, mouse, worm, and fruit fly indicated no correlation between $\Sigma$ RAS versus $\Sigma$ RASGEF and also not between $\Sigma$ RAS and $\Sigma$ RASGAP. However, similar to the gene expression analysis, reasonable $\Sigma$ RASGEF versus $\Sigma$ RASGAP correlation values were observed for three organisms, but not for worm (Additional file 2). We further analysed the protein expression levels across human tissues (PaxDB [26] and Kim et al. [3]), where generally good correlation values between $\Sigma$ RASGEF versus $\Sigma$ RASGAP were found (Fig. 1e; Additional file 2). The correlation values of $\Sigma$ RASGEF versus $\Sigma$ RASGAP for protein and gene expression levels were in good agreement (Fig. 1f) even though not perfect - due to the low abundance of proteins. In conclusion, the overall correlation of $\Sigma$ RASGEF and $\Sigma$ RASGAP protein/gene levels in normal tissues indicates a balance of these entities.

\section{Correlation of $\Sigma R A S$, ERASGEF and ERASGAP levels in adult normal tissues across individuals}

As the gene expression values from the human normal tissues from the GTEx consortium were average values of several individuals for each tissue (dataset $h s$ NT_03), we next analysed whether or not the expression balance was even stronger in the same tissues of different individuals. In the small intestine tissue, for example, the $\Sigma$ GEF and $\Sigma$ GAP levels spanned a large expression range from 100 to 500 RPKM in different individuals, but the $\Sigma$ GEF and $\Sigma$ GAP levels were balanced in the same person (Fig. 2a). In other tissues that had a smaller dynamic range of expression (e.g. colon) we still found a significant correlation (Fig. 2b). We observed this expression balance throughout all/most tissues (Fig. 2c, Additional file 1: Figure S5, and Additional file 6). As observed before using the averaged tissue expression datasets, the correlation between RAS members and their GEFs or GAPs was generally lower 
a

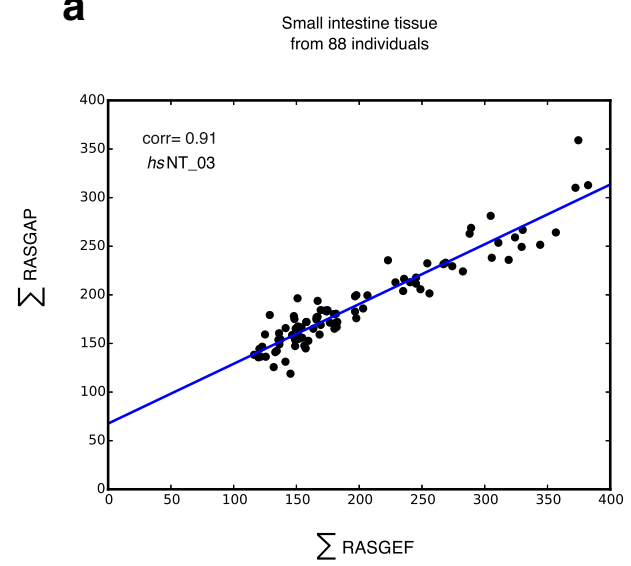

b

Colon tissue

from 345 individuals

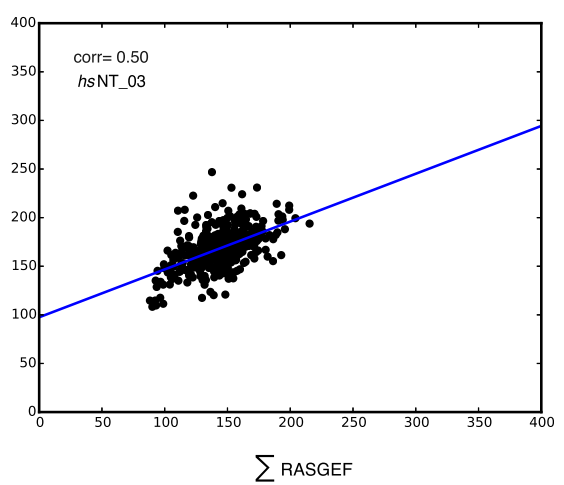

C

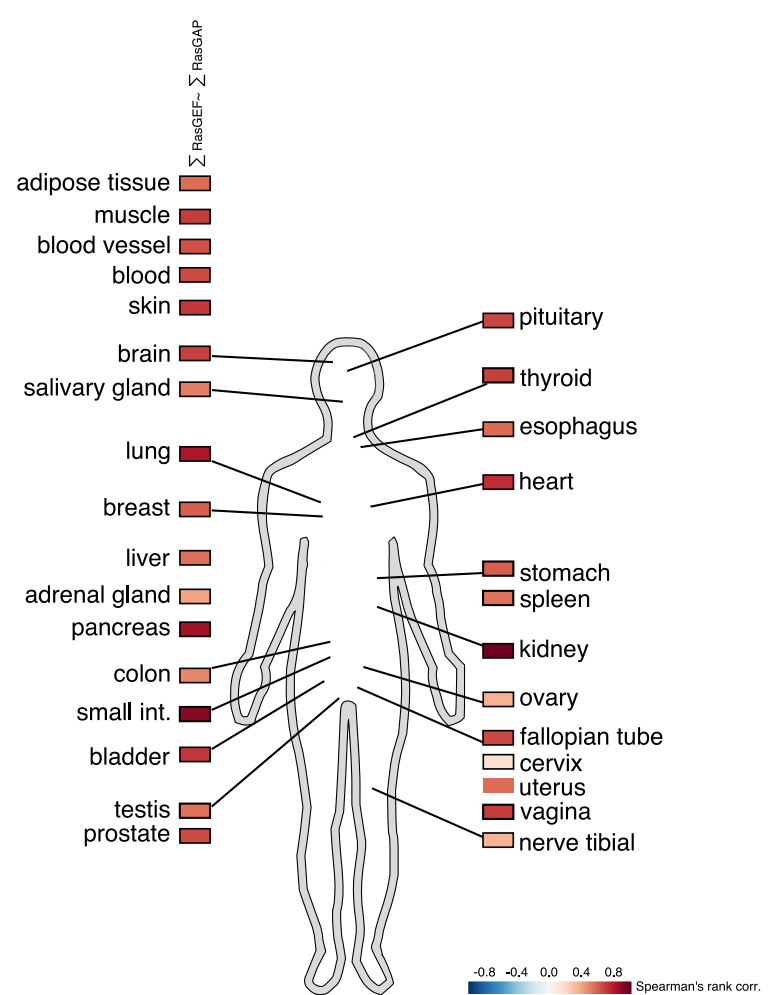

Fig. 2 Spearman's rank correlations in different tissues across individuals. a Correlation of the $\sum G E F$ with the $\sum G A P$ in small intestine across 88 individuals (GTEx, hsNT_03). b Correlation of the $\sum$ GEF with the $\sum$ GAP in colon across 149 individuals (GTEx, hsNT_03). c Heatmap of Spearman'rank correlation values across individuals for all tissue analysed. The part of the picture that represents a scheme of a human being was drawn using Inkscape

(Additional file 6). In summary, the expression levels of GEF and GAP family members may vary in different individuals for a particular tissue, but the sum of all GEFs and GAPs was found to correlate in the same person.

\section{Gene expression ratios of RAS and regulators in normal adult tissues}

To obtain insights into the quantitative relation among RAS and regulators, we compared the ratios of the $\Sigma$ RAS, $\Sigma$ GEF, and $\Sigma$ GAP levels across the human normal tissues.
We observed that the ratios for a given tissue were quite comparable in various datasets (Additional file 7; Additional file 1: Figure S6). We found that the average $\Sigma$ GEF/ $\Sigma$ GAP ratio across all tissues was 1.12 , but ratios could be as low as 0.69 and as high as 2.57 , suggesting that the ratio is constant in a particular tissue (Fig. 3). However, we could not identify a correlation between the ratio and tissue turnover times as a measure of tissue proliferation [29], suggesting that the $\Sigma$ GEF/ $/$ GAP ratio in individual tissues is unrelated to proliferation (Additional file 7). 


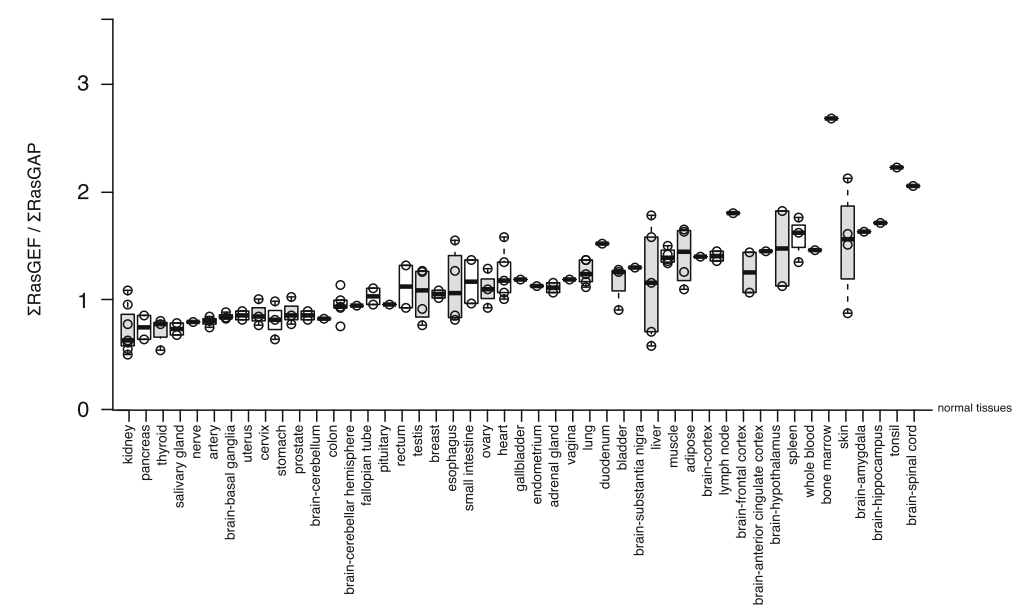

Fig. 3 Ratios for the sum of RasGEF and RasGAP regulators in normal tissues. a Analysis of $\sum$ RasGEF/ $\Sigma$ RasGAP ratios in different human normal tissues (datasets hsNT_01, hsNT_02, hsNT_03, hsNT_04, and hsNT05). Similar tissues from different datasets were averaged (see Additional file 7)

\section{Loss of GEF and GAP expression correlation in human cancer tissues and cell lines}

Correlation and clustering signatures previously revealed that various cancer types share a common gene expression profile, which differs from normal tissues and suggesting an underlying 'near universal' cellular dysfunction that is associated with cancer [30, 31]. Thus, we analysed the correlations between the $\Sigma$ GEFs and $\Sigma$ GAPs using cancer tissue expression datasets (datasets $h s \mathrm{CT}$ 01, hsCT_02, and $h s \mathrm{CT}$ _03) and cell lines (dataset hsCL_01 and hsCL_02) (Additional file 8). We found that the correlation value was often lower or lost in cancer tissues and cell lines (Additional file 1: Figure S7). Moreover, the correlation values for the cancer datasets were often not significantly different from the randomized data (Additional file 9). This suggests that some cancer tissues and cell lines do not have balanced $\Sigma$ GEF and $\Sigma$ GAP levels.

To obtain further insights into the loss of correlation, we next analysed correlations across individuals in different cancer tissues (from the TCGA database). The TCGA data contain corresponding 'normal' tissue data that were taken from the same tissue, but from areas further away from the tumour (datasets $h s \mathrm{CT} \_01$ and $h s \mathrm{NT}$ 04). As the number of samples with from those 'normal' individual tissues were generally lower, we also analysed the normal tissues from individuals from the GTEx database ( $h s$ NT_03). The normal tissues from TCGA ( $h s \mathrm{NT}$-04) and GTEx ( $h s$ NT_03) generally agreed well when comparing the same tissue (Additional file 1: Figure S8; Additional file 10). For each tissue we performed correlations across individuals by treating the merged normal tissue data ( $h s \mathrm{NT}_{0} 03$ and $h s \mathrm{NT} \_04$ ) and cancer tissue data separately (Fig. 4; Additional file 1: Figure S8). Similarly to the overall correlations across cancer tissues, we observed that generally the correlations for specific cancer tissues were lower compared to normal tissues (Fig. 4; Additional file 1: Figure S8; Additional file 11) (16\% of matching data and $23 \%$ of merged data had a significant change in correlation, $p$-value $<0.05)$. More importantly, however, we found that the slopes of $\Sigma$ RASGEF- 2 RASGAP were in five cases (bladder, liver, ovary, prostate, stomach) significantly increased, p-value 0.05 (Fig. 4; Additional file 1: Figure S8; Additional file 11). The increased slopes were due to increased $\Sigma$ RASGAP levels in some tumours. However, there was a large heterogeneity in the tumours and only a fraction of tumours had higher $\Sigma$ RASGAP levels. While there was no relation observed between the $\Sigma \mathrm{GAP} / \Sigma \mathrm{GEF}$ ratios and tumour stages (Additional file 12), for some cancer tissues (lung, cervix, and liver), a significant decrease in patient survival was observed for higher $\Sigma G A P / \Sigma G E F$ ratios suggesting that this ratio may be used as a prognostic indicator for survival (Additional file 1: Figure S9).

Taken together, these data suggest that the expression levels of small Ras-like GTPase regulators must be balanced to achieve the normal physiological functioning. The dysregulation of $\Sigma$ GEF, $\Sigma$ GAP and $\Sigma$ RAS levels suggest that this may serve an additional hallmark of some cancers.

\section{Discussion}

Here we show that sum of gene expression levels of GEF and GAP regulators of RAS GTPases is balanced in physiologically normal cell types and tissues. Genetic buffering and compensation has been shown to involve paralogs of genes that contain both functional overlap and functional differences [32]. The functional overlap for the families of GEFs or GAPs is given by their catalytic domains with GEF or GAP activity towards RAS small 

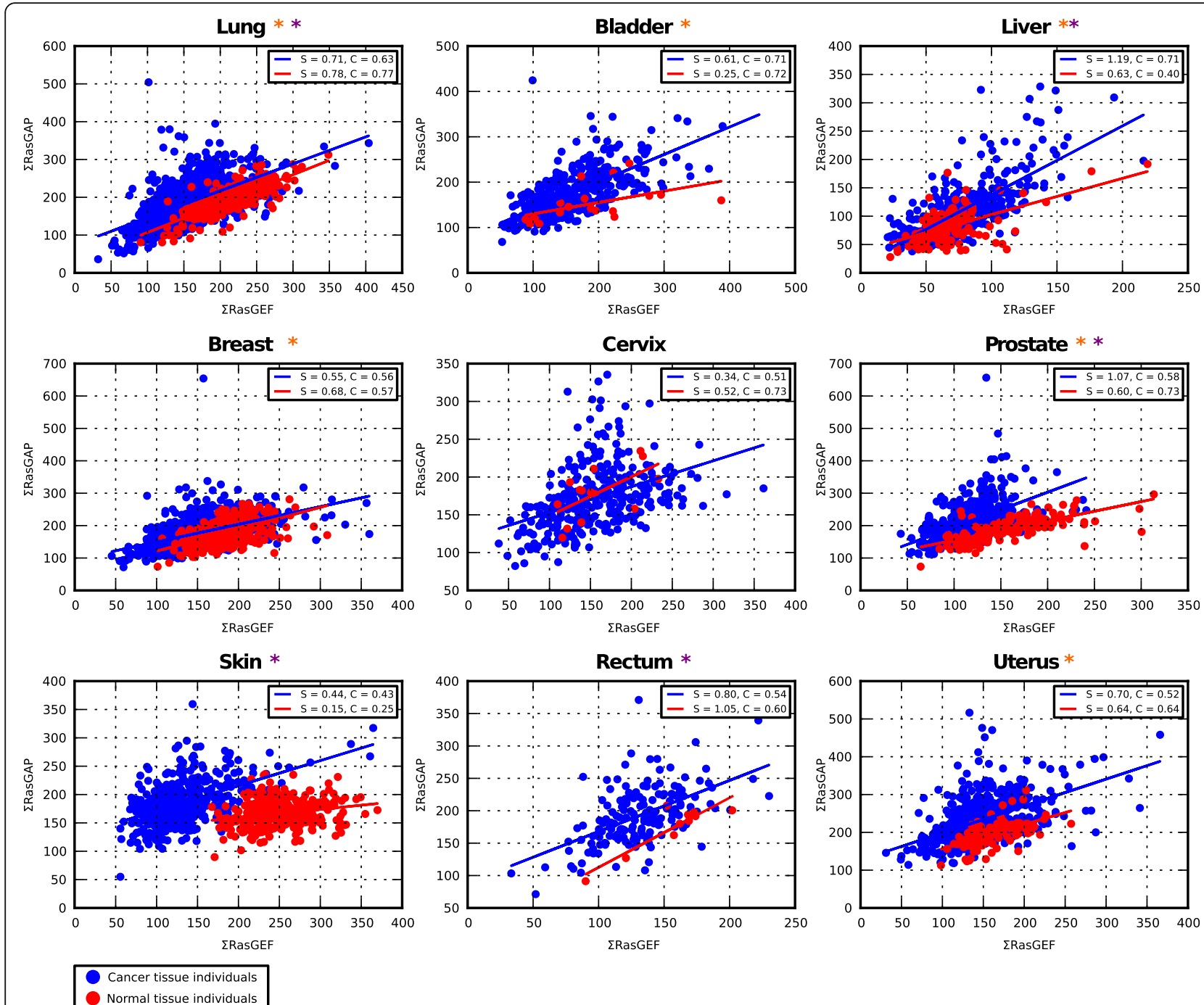

Fig. 4 Correlations of the sum of RasGEF and RasGAP across individuals in different tissues. Spearman's rank correlation of the $\sum$ RasGEF with the ¿RasGAP in different normal (red squares) and cancer (blue squares) tissues across individuals (datasets hsNT_03 and hsCT_01). The correlation values $(C)$ and slopes (S) are indicated in the figure. Significantly different changes in slopes or correlations comparing normal and cancer tissues are indicated next to the tissue name (plot titles), with orange and purple stars $\left(^{*}\right)$, respectively

GTPases. The functional differences can be achieved using their multidomain nature containing a wide-range of different domains, which can affect their localization or specific functionalities such as the formation of complexes with other cellular proteins. Thus, individual activating (GEFs) and deactivating domains (GAPs) could change significantly their expression to perform particular functions using their other domains in particular tissues, but the overall balance for the GEF and GAP functionality is kept. The balanced expression of GEFs and GAPs should in principle enable a constant RAS activity (GTP vs GDP bound forms) in the normal tissues under constant growth signals, although protein levels do not necessarily relate 1:1 to protein activities. The finding that a subset of genes correlates weaker than the complete set but stronger than the random set is not unexpected. It is possible that, in future work, the identification of other subsets that correlate and characterizing their functions experimentally will further our understanding of the mechanisms of control of GEF/GAP expression and their role in the Ras pathway.

We also show that the GEF-GAP expression correlation is lost in some individual tumour tissues and cell lines. GEFs and GAPs have been frequently found to be differentially expressed in cancer [15, 33, 34]. Correlation and clustering signatures previously revealed that various cancer types share a common gene expression profile, which differs from normal tissues and suggests an underlying 'near universal' cellular dysfunction that results in cancer [30,31]. Our work suggests that imbalances of GEF and GAP family members could be one 
feature of this dysregulation in some cancers. However, in is unclear if it is a cause or consequence of cancer development.

The fundamental mechanistic question of how levels of GEF and GAP are regulated and why they are altered in cancer tissues warrants further investigation. It is expected that the dosage of genes belonging to a functional group are under the control of signal transduction pathways and transcription factors [35]. For example, it has been shown that polo-like kinase 2 coordinates multiple GAPs and GEFs for synchronised tuning and activation of Ras and Rap small GTPases important for synaptic plasticity [36]. The most likely explanation being that the RAS activity state and downstream signalling itself induces positive and negative feedback mechanisms that control GEF and GAP levels through transcriptional regulation. Therein, a likely hypothesis is that levels are regulated by both GEF- and GAP-specific transcription factors and general transcription factors induced downstream of RAS.

It is quite remarkable that there are large differences in the sum of GEF and sum of GAP levels in the same tissue for different individuals, while in each individual a near perfect balance of sum of GEFs and GAPs is found. These individual expression differences are important to be considered in personalised medicine approaches, where for example a marker GEF protein that is highly expressed might be indicative of a bad prognosis in some individuals. However, in those individuals that have in general lower GEF and GAP levels this marker GEF protein might be missed if one compares to the average population expression levels.

Taken together, our data suggest that the expression levels of regulators of RAS GTPases must be balanced to achieve the normal physiological function in adult tissues. It is quite likely that similar phenomena are found in other multidomain groups of proteins sharing a functional domain. Thus, the finding that the protein paralogue expression levels quite often anticorrelate to keep constant a certain common functionality must now be extended to proteins sharing a common functional domain like GEFs and GAPs. For example, we have shown in mouse small intestine that knocking out the RhoGEF Tiam1 or Vav3 causes the upregulation of another RhoGEF, Vav2 (Pickering et al., manuscript under revision in Nature Communications). Only by knocking out all the three RhoGEFs simultaneously is suppression of the $\mathrm{APC}^{\mathrm{fl} / \mathrm{fl}}$ hyperproliferative phenotype observed.

\section{Conclusions}

Our study shown that the function of multidomain proteins cannot be understood without considering all family homologs and explains why in many cases homologs exhibit expression anticorrelation. We suggest that future experimental analyses should include systemic changes of all protein family members rather than focusing on one particular protein. Further, our results are relevant for critical considerations of knock out experiments, where functionally related homologs may compensate for the down regulation of a protein.

\section{Additional files}

Additional file 1: Figure S1. The Ras superfamily and regulators. Figure S2. Gene expression datasets analysed in this study. Figure S3. Example of sum and pairwise correlations. Figure S4. Correlations across adult normal tissues. Figure S5. Correlations across individuals in the background of random correlations. Figure $\mathbf{S 6}$. Example ratios in human lung tissues. Figure S7. Correlations in cancer tissues and cell lines. Figure S8. Correlations across individuals in normal and cancer tissues. Figure S9. Survival analysis of individuals with high and low RasGAP/ RasGEF ratios. (DOCX 1465 kb)

Additional file 2: Protein expression levels and correlations in organisms and normal tissues. (XLSX $19 \mathrm{~kb}$ )

Additional file 3: RAS members and GEF and GAP regulators. (XLSX 83 kb)

Additional file 4: Ratios and correlations in human adult tissues. (XLSX $34 \mathrm{~kb}$ )

Additional file 5: Gene expression correlations for random shuffled genes in adult tissues. (XLSX 8257 kb)

Additional file 6: Gene expression correlations across human individuals in normal adult tissues. (XLSX 12 kb)

Additional file 7: Classification of human normal tissues into groups of similar tissues, ratio of sum of RASGEF to sum of RASGAP ratios, and tissue turnover times. (XLSX $13 \mathrm{~kb}$ )

Additional file 8: Ratios and correlations of gene expression levels in cancer tissues and cell lines. (XLSX $41 \mathrm{~kb}$ )

Additional file 9: Gene expression correlations for random shuffled genes in cancer tissues and cell lines. (XLSX 613 kb)

Additional file 10: Sum of RASGEF versus sum of RASGAP gene expression levels for human individuals in adult tissues and cancer tissues. (XLSX $296 \mathrm{~kb}$ )

Additional file 11: Slopes and correlations of sum of RASGEF versus sum of RASGAP gene expression levels in normal versus cancer tissues. (XLSX $14 \mathrm{~kb}$ )

Additional file 12: Gene expression correlations in cancer tissues according to tumor stages. (XLSX $11 \mathrm{~kb}$ )

\section{Abbreviations}

COSMIC: Catalogue of somatic mutations in cancer; GAP: GTPase activating protein; GEF: Guanine nucleotide exchange factor; GTPase: Family of hydrolase enzymes that can bind and hydrolyse guanosine triphosphate (GTP); RPKM: Reads per kilobase per million mapped reads; TCGA: The cancer genome atlas; TPM: Transcripts per kilobase million

\section{Acknowledgements}

We thank Eren Naz Dogan for help in the design of figures (https:// www.behance.net/erennazunal).

Ethical approval and consent to participate Not applicable.

\section{Availability of data and materials}

All data generated or analysed during this study are included in this published article [and its supplementary information files] or are publicly available. 


\section{Funding}

This work was funded by the EU (PRIMES under grant agreement number FP7-HEALTH-F4- 2011-278568). This work was supported by the Spanish Ministerio de Economía y Competitividad, Plan Nacional BIO2012-39754 and Plan Estatal BFU2015-63571 and the European Fund for Regional Development. We acknowledge support of the Spanish Ministry of Economy, Industry and Competitiveness (MEIC) to the EMBL partnership, to the Spanish Ministry of Economy and Competitiveness (MEC) 'Centro de Excelencia Severo Ochoa' and to the CERCA Programme / Generalitat de Catalunya.

\section{Authors' contributions}

$E B U, C K, H B, A C$, and KP analysed data. EBU, CK, NB, OJS, and LS contributed to the study design. EBU, CK, and LS wrote the paper with input from all other authors. All authors read and approved the final manscript.

\section{Consent for publication}

Not applicable.

\section{Competing interests}

The authors declare that they have no competing interests.

\section{Publisher's Note}

Springer Nature remains neutral with regard to jurisdictional claims in published maps and institutional affiliations.

\section{Author details}

'Institute of Pathology, Charité - Universitätsmedizin Berlin, 10117 Berlin, Germany. ${ }^{2}$ Integrative Research Institute Life Sciences, Humboldt Universität Berlin, 10115 Berlin, Germany. ${ }^{3}$ Centre for Genomic Regulation (CRG), Systems Biology Programme. The Barcelona Institute of Science and Technology, Dr. Aiguader 88, Barcelona 08003, Spain. ${ }^{4}$ Present address: Systems Biology Ireland \& Charles Institute of Dermatology \& School of Medicine, University College Dublin, Belfield, Dublin 4, Ireland. ${ }^{5}$ Cancer Research UK Beatson Institute, Garscube Estate, Switchback Road, Glasgow G61 1BD, UK. ' Universitat Pompeu Fabra (UPF), 08003 Barcelona, Spain. ${ }^{7}$ Institució Catalana de Recerca i Estudis Avançats (ICREA), Pg. Lluís Companys 23, 08010 Barcelona, Spain.

Received: 21 March 2018 Accepted: 2 August 2018 Published online: 15 August 2018

\section{References}

1. Uhlén M, Fagerberg L, Hallström BM, Lindskog C, Oksvold P, Mardinoglu A, Sivertsson Å, Kampf C, Sjöstedt E, Asplund A, Olsson I, Edlund K, Lundberg E, Navani S, Szigyarto CA, Odeberg J, Djureinovic D, Takanen JO, Hober S, Alm T, et al. Proteomics. Tissue-based map of the human proteome Science. 2015:347(1260419)

2. Geiger T, Velic A, Macek B, Lundberg E, Kampf C, Nagaraj N, Uhlen M, Cox J, Mann M. Initial quantitative proteomic map of 28 mouse tissues using the SILAC mouse. Mol Cell Proteomics. 2013;12:1709-22.

3. Kim MS, Pinto SM, Getnet D, Nirujogi RS, Manda SS, Chaerkady R, Madugundu AK, Kelkar DS, Isserlin R, Jain S, Thomas JK, Muthusamy B, LealRojas P, Kumar P, Sahasrabuddhe NA, Balakrishnan L, Advani J, George B, Renuse $\mathrm{S}$, Selvan LD, et al. A draft map of the human proteome. Nature. 2014;509:575-81.

4. Wilhelm M, Schlegl J, Hahne H, Gholami AM, Lieberenz M, Savitski MM, Ziegler E, Butzmann L, Gessulat S, Marx H, Mathieson T, Lemeer S, Schnatbaum K, Reimer U, Wenschuh H, Mollenhauer M, Slotta-Huspenina J, Boese JH, Bantscheff M, Gerstmair A, et al. Mass-spectrometry-based draft of the human proteome. Nature. 2014;509:582-7.

5. Weiss M, Schrimpf S, Hengartner MO, Lercher MJ, von Mering C. Shotgun proteomics data from multiple organisms reveals remarkable quantitative conservation of the eukaryotic core proteome. Proteomics. 2010;10:1297-306.

6. Chan ET, Quon GT, Chua G, Babak T, Trochesset M, Zirngibl RA, Aubin J, Ratcliffe MJ, Wilde A, Brudno M, Morris QD, Hughes TR. Conservation of core gene expression in vertebrate tissues. J Biol. 2009;8:33.

7. Chandra A, Grecco HE, Pisupati V, Perera D, Cassidy L, Skoulidis F, Ismail SA, Hedberg C, Hanzal-Bayer M, Venkitaraman AR, Wittinghofer A, Bastiaens PI. The GDI-like solubilizing factor PDEdelta sustains the spatial organization and signalling of Ras family proteins. Nature Cell Biol. 2012;14:148-58.
8. Assis R, Bachtrog D. Rapid divergence and diversification of mammalian duplicate gene functions. BMC Evol Biol. 2015;15:138.

9. Kryuchkova-Mostacci N, Robinson-Rechavi M. Tissue-specificity of gene expression diverges slowly between Orthologs, and rapidly between paralogs. PLoS Comput Biol. 2016;12:e1005274.

10. Toufighi K, Yang JS, Luis NM, Aznar Benitah S, Lehner B, Serrano L, Kiel C. Dissecting the calcium-induced differentiation of human primary keratinocytes stem cells by integrative and structural network analyses. PLoS Comput. Biol. 2015;11:e1004256.

11. Kirsch L, Chechik G. On expression patterns and developmental origin of human brain regions. PLoS Comput Biol. 2016;12:e1005064.

12. Rojas AM, Fuentes $G$, Rausell A, Valencia A. The Ras protein superfamily: evolutionary tree and role of conserved amino acids. J Cell Biol. 2012;196:189-201.

13. Maertens $\mathrm{O}$, Cichowski K. An expanding role for RAS GTPase activating proteins (RAS GAPs) in cancer. Adv Biol Regul. 2014:55:1-14.

14. Vetter $\mathbb{R}$, Wittinghofer $A$. The guanine nucleotide-binding switch in three dimensions. Science. 2001:294:1299-304.

15. Vigil D, Cherfils J, Rossman KL, Der CJ. Ras superfamily GEFs and GAPs: validated and tractable targets for cancer therapy? Nat Rev Cancer. 2010;10:842-57.

16. Cherfils J, Zeghouf M. Regulation of small GTPases by GEFs, GAPs, and GDIs. Physiol Rev. 2013:93:269-309.

17. Bos JL, Rehmann H, Wittinghofer A. GEFs and GAPs: critical elements in the control of small G proteins. Cell. 2007:129:865-77.

18. Krupp M, Marquardt JU, Sahin U, Galle PR, Castle J, Teufel A. RNA-Seq atlas-a reference database for gene expression profiling in normal tissue by nextgeneration sequencing. Bioinformatics. 2012;28:1184-5.

19. GTEx Consortium. The Genotype-Tissue Expression (GTEx) project. Nat. Genet 2013:45:580-5.

20. Human genomics. The Genotype-Tissue Expression (GTEx) pilot analysis: multitissue gene regulation in humans. Science. 2015;348:648-60.

21. Brawand D, Soumillon M, Necsulea A, Julien P, Csárdi G, Harrigan P, Weier M, Liechti A, Aximu-Petri A, Kircher M, Albert FW, Zeller U, Khaitovich P, Grützner F, Bergmann S, Nielsen R, Pääbo S, Kaessmann H. The evolution of gene expression levels in mammalian organs. Nature. 2011;478:343-8.

22. Su Al, Cooke MP, Ching KA, Hakak Y, Walker JR, Wiltshire T, Orth AP, Vega RG, Sapinoso LM, Mogrich A, Patapoutian A, Hampton GM, Schultz PG, Hogenesch JB. Large-scale analysis of the human and mouse transcriptomes. Proc Natl Acad Sci U S A. 2002:99:4465-70.

23. Chintapalli VR, Wang J, Dow JA. Using FlyAtlas to identify better Drosophila melanogaster models of human disease. Nat Genet. 2007:39:715-20.

24. Forbes $S A$, Beare D, Gunasekaran $P$, Leung K, Bindal N, Boutselakis H, Ding M, Bamford S, Cole C, Ward S, Kok CY, Jia M, De T, Teague JW, Stratton MR, McDermott U, Campbell PJ. COSMIC: exploring the world's knowledge of somatic mutations in human cancer. Nucleic Acids Res. 2015;43:D805-11.

25. Barretina J, Caponigro G, Stransky N, Venkatesan K, Margolin AA, Kim S, Wilson CJ, Lehár J, Kryukov GV, Sonkin D, Reddy A, Liu M, Murray L, Berger MF, Monahan JE, Morais P, Meltzer J, Korejwa A, Jané-Valbuena J, Mapa FA, et al. The Cancer cell line encyclopedia enables predictive modelling of anticancer drug sensitivity. Nature. 2012;483:603-7.

26. Wang $M$, Weiss $M$, Simonovic $M$, Haertinger $G$, Schrimpf SP, Hengartner MO, von Mering C. PaxDb, a database of protein abundance averages across all three domains of life. Mol Cell Proteomics. 2012;11:492-500.

27. Ezkurdia I, Vazquez J, Valencia A, Tress M. Analyzing the First Drafts of the Human Proteome: J. Proteome Res; 2014.

28. Guo Y, Sheng Q, Li J, Ye F, Samuels DC, Shyr Y. Large scale comparison of gene expression levels by microarrays and RNAseq using TCGA data. PLoS One. 2013;8:e71462.

29. Richardson RB, Allan DS, Le Y. Greater organ involution in highly proliferative tissues associated with the early onset and acceleration of ageing in humans. Exp Gerontol. 2014;55:80-91.

30. Yap Y, Zhang X, Ling MT, Wang X, Wong YC, Danchin A. Classification between normal and tumor tissues based on the pair-wise gene expression ratio. BMC Cancer. 2004:4:72

31. Slavov N, Dawson KA. Correlation signature of the macroscopic states of the gene regulatory network in cancer. Proc Natl Acad Sci U S A. 2009:106:4079-84

32. Diss G, Ascencio D, DeLuna A, Landry CR. Molecular mechanisms of paralogous compensation and the robustness of cellular networks. J Exp Zool B Mol Dev Evol. 2014;322:488-99.

33. Grewal T, Koese M, Tebar F, Enrich C. Differential regulation of RasGAPs in Cancer. Genes Cancer. 2011;2:288-97. 
34. Ksionda O, Limnander A, Roose JP. RasGRP Ras guanine nucleotide exchange factors in cancer. Front Biol. 2013;8:508-32.

35. Birchler JA, Veitia RA. The gene balance hypothesis: from classical genetics to modern genomics. Plant Cell. 2007;19:395-402.

36. Lee K, Lee Y, Rozeboom A, Lee JY, Udagawa N, Hoe HS, Pak DT.

Requirement for PIk2 in orchestrated ras and rap signaling, homeostatic structural plasticity, and memory. Neuron. 2011;69:957-73.

Ready to submit your research? Choose BMC and benefit from:

- fast, convenient online submission

- thorough peer review by experienced researchers in your field

- rapid publication on acceptance

- support for research data, including large and complex data types

- gold Open Access which fosters wider collaboration and increased citations

- maximum visibility for your research: over $100 \mathrm{M}$ website views per year

At $\mathrm{BMC}$, research is always in progress.

Learn more biomedcentral.com/submissions 